\title{
Development of a whole-cell biocatalyst for diisobutyl phthalate degradation by functional display of a carboxylesterase on the surface of Escherichia coli
}

Junmei Ding ${ }^{1,2,3^{*}+} \oplus$, Yang Zhou ${ }^{1,2,3 \dagger}$, Chaofan Wang ${ }^{1,2,3 \dagger}$, Zheng Peng ${ }^{1,2,3}$, Yuelin Mu ${ }^{1,2,3}$, Xianghua Tang ${ }^{1,2,3}$ and Zunxi Huang ${ }^{1,2,3^{*}}$

\begin{abstract}
Background: Phthalic acid esters (PAEs) are widely used as plasticizers or additives during the industrial manufacturing of plastic products. PAEs have been detected in both aquatic and terrestrial environments due to their overuse. Exposure of PAEs results in human health concerns and environmental pollution. Diisobutyl phthalate is one of the main plasticizers in PAEs. Cell surface display of recombinant proteins has become a powerful tool for biotechnology applications. In this current study, a carboxylesterase was displayed on the surface of Escherichia coli cells, for use as whole-cell biocatalyst in diisobutyl phthalate biodegradation.

Results: A carboxylesterase-encoding gene (carEW) identified from Bacillus sp. K91, was fused to the N-terminal of ice nucleation protein (inpn) anchor from Pseudomonas syringae and gfp gene, and the fused protein was then cloned into pET-28a(+) vector and was expressed in Escherichia coli BL21(DE3) cells. The surface localization of INPN-CarEW/ or INPN-CarEW-GFP fusion protein was confirmed by SDS-PAGE, western blot, proteinase accessibility assay, and green fluorescence measurement. The catalytic activity of the constructed E. coli surface-displayed cells was determined. The cell-surface-displayed CarEW displayed optimal temperature of $45^{\circ} \mathrm{C}$ and optimal pH of 9.0, using p-NPC as substrate. In addition, the whole cell biocatalyst retained $\sim 100 \%$ and $\sim 200 \%$ of its original activity per $\mathrm{OD}_{600}$ over a period of 23 days at $45^{\circ} \mathrm{C}$ and one month at $4{ }^{\circ} \mathrm{C}$, exhibiting the better stability than free CarEW. Furthermore, approximately $1.5 \mathrm{mg} / \mathrm{ml}$ of DiBP was degraded by $10 \mathrm{U}$ of surface-displayed CarEW cells in $120 \mathrm{~min}$.
\end{abstract}

Conclusions: This work provides a promising strategy of cost-efficient biodegradation of diisobutyl phthalate for environmental bioremediation by displaying CarEW on the surface of E. coli cells. This approach might also provide a reference in treatment of other different kinds of environmental pollutants by displaying the enzyme of interest on the cell surface of a harmless microorganism.

Keywords: Cell surface display, Phthalic acid esters, Whole-cell biocatalyst, Diisobutyl phthalate, Carboxylesterase

*Correspondence: djm3417@163.com; huangzunxi@163.com

†Junmei Ding, Yang Zhou and Chaofan Wang to be considered as joint

first authors

${ }^{1}$ Engineering Research Center of Sustainable Development

and Utilization of Biomass Energy, Ministry of Education, Yunnan Normal University, Kunming 650500, Yunnan, China

Full list of author information is available at the end of the article

\section{Background}

Phthalic acid esters (PAEs) are a class of organic compounds that are widely used as plasticizers or additives during the industrial manufacturing of plastic products [1]. PAEs provide flexibility, durability, and elasticity to polyvinyl chloride (PVC) resins and other polymers by physically interacting with polymeric matrices which

(c) The Author(s) 2020. This article is licensed under a Creative Commons Attribution 4.0 International License, which permits use, sharing, adaptation, distribution and reproduction in any medium or format, as long as you give appropriate credit to the original author(s) and the source, provide a link to the Creative Commons licence, and indicate if changes were made. The images or other third party material in this article are included in the article's Creative Commons licence, unless indicated otherwise in a credit line to the material. If material is not included in the article's Creative Commons licence and your intended use is not permitted by statutory regulation or exceeds the permitted use, you will need to obtain permission directly from the copyright holder. To view a copy of this licence, visit http://creativeco mmons.org/licenses/by/4.0/. The Creative Commons Public Domain Dedication waiver (http://creativecommons.org/publicdomain/ zero/1.0/) applies to the data made available in this article, unless otherwise stated in a credit line to the data. 
make PAEs directly and/or indirectly migrating into the environment during industrial production, utilization, or disposal. Consequently, PAEs are detected not only in aquatic but also in terrestrial environments [2]. However, after over a half century of supposedly safe utilization, several experimental studies have demonstrated that PAEs could cause adverse effects to human health [3], including disrupt the endocrine systems [4], induce reproductive toxicity [5] and hepatocellular tumors, harm fetal health $[3,6]$, and so on. As one of the main plasticizers in PAEs, diisobutyl phthalate (DiBP) has permanently been banned by the U.S. Consumer Products Safety Commission (CPSC) because of its reproductive toxicity [7]. Therefore, the perception of environmental and health risks imposed by PAEs has fundamentally changed and important issues pertaining to the environmental fate of PAEs have been raised.

As sustainable and hazardous contamination of PAEs to environment and our human health, biodegradation of PAEs in the environment attracted more attention [1, $2,6]$. Microbial degradation especially bacteria-mediated biodegradation is considered the most promising method for removing PAEs from polluted environments. Several microorganisms and their related critical enzymes capable of degrading PAEs were summarized in Table 1, bacteria from genus of Sphingobium [8], Pseudomonas [9], Bacillus [10], Sulfobacillus [11], Acinetobacter [12], Rhodococcus [13], Fusarium [14, 15], Gordonia [16], and Micrococcus [17] were included. Moreover, esterases from tissues [18] or uncultured microorganisms [19] with PAEs biodegradation ability were also reported. Based on the identification of associated metabolic intermediates, two steps are involved in the metabolic pathways associated with PAE biodegradation: (i) transformation of PAEs to phthalic acid (PTH) and (ii) complete degradation of
PTH. Esterases/hydrolases expressed by microorganisms played critical role in both steps [20]. However, until now, only few esterases/hydrolases involved in PAE decomposition have been characterized.

Carboxylesterases (EC 3.1.1.1), also known as esterases, are widely distributed in nature and play multiple important functions in the detoxification of various harmful exogenous compounds, such as herbicides [21], pesticides [22], and so on. With a catalytic triad composed of Ser-Asp (or Glu)-His and a consensus sequence (G-X-S-X-G) around the active site serine residues, carboxylesterases belong to the $\alpha / \beta$ hydrolase superfamily and catalyze the hydrolysis of carboxylic ester bonds $(<10$ carbon atoms). Together with lipases, both are very important industrial enzymes and are widely distributed in nature [23]. Carboxylesterases or lipases exhibit stable thermostability, accept wide range of substrates, require no cofactor, maintain high regio/stereo-specificity, remain stable in organic solvents. These properties make carboxylesterases or lipases to be used as biocatalysts in a variety of industrial processes, including biochemical, food, pharmaceutical, and biological purposes [24]. However, the purification costs, low catalytic activities and poor enzyme stability of the requisite enzymes are all concerns for large scale practical applications.

Many of these problems mentioned above can be solved by displaying useful foreign enzymes on live microbial cell surface by fusing them with appropriate anchoring motifs. Anchorage of target enzymes on the outer membrane of model microorganisms allows direct enzymatic reaction with substrates with no need of crossing the membrane barrier and purifying the enzymes which significantly reduce the cost of whole cell biocatalyst preparation and application [25]. Previous investigations showed that the microbial surface display systems

Table 1 Bacteria and related esterases involved in PAEs biodegradation

\begin{tabular}{llll}
\hline Esterases & Sources & Substrates & References \\
\hline Carboxylesterase & Sphingobium yanoikuyae & Dinbutyl phthalate & {$[8]$} \\
Hydrolase & Pseudomonas mosselii & Mono-2-ethylhexyl phthalate & {$[9]$} \\
CarEW & Bacillus sp. K91 & Diisobutyl phthalate & {$[10]$} \\
EstS1 & Sulfobacillus acidophilus & Phthalate esters & {$[11]$} \\
PE hydrolase & Acinetobacter sp. M673 & Dibutyl phthalate & Monoalkyl phthalate \\
PatE & Rhodococcus jostii RHA1 & Dimethyl terephthalate & {$[12]$} \\
DMT esterase & Fusarium sp. DMT-5-3 & Phthalate esters & {$[13]$} \\
Cutinase & Fusarium oxysporum & Mono-2-ethylhexyl Phthalate & {$[14]$} \\
Esterase & Gordonia sp. P8219 & Monoalkyl phthalates & {$[16]$} \\
Esterases & Micrococcus sp. YGJ1 & Phthalate esters & {$[17]$} \\
Esterase & Pancreatic cholesterol & Dibutyl phthalate & {$[18]$} \\
DphB & Metagenomics library & {$[19]$} \\
\hline
\end{tabular}


have been successfully applied in various fields, including food industry [26], bioremediation [27], biofuel [28], biological synthesis [29], and so on. Among the anchoring motifs, the truncated $\mathrm{N}$-terminal domain of ice nucleation protein (INP) identified from Pseudomonas syringae has been proven to be an efficient carrier [25]. However, the INP-mediated surface display method has not been used till now for the PAEs biodegradation although various successful applications of INP-anchored functional proteins have been reported.

In this study, a carboxylesterase, CarEW, was identified from Bacillus sp. K91 and functionally displayed on the surface of $E$. coli cells by fusing CarEW with the INPN anchoring motif. The environmentally friendly engineered $E$. coli strain was endowed with the capacity to degrade PAEs and could be potentially used for further environmental bioremediation. Additionally, this study may also provide a method for the biodegradation of other environmental pollutants.

\section{Results}

\section{Expression of CarEW/GFP and INPN/CarEW/GFP fusion proteins}

The CarEW encoding gene carEW with a 1464-long ORF was amplified from Bacillus sp. K91 and the recombinant E. coli strain BL21(DE3)-pEASY-E2/carEW was constructed previously in our lab [10]. CarEW was composed of 487 amino acids and had a molecular mass of approximately $53.76 \mathrm{kDa}$ with a $\mathrm{pI}$ of 4.88 . Sequence alignment showed that CarEW shares less than $37 \%$ sequence similarity with some reported esterases which were capable of degrading PAEs (Fig. 1).

In this study, recombinant plasmids pET$28 \mathrm{a}(+) / c a r E W, \quad \mathrm{pET}-28 \mathrm{a}(+) / c a r E W / g f p$, and $\mathrm{pET}-$ $28 \mathrm{a}(+) /$ inpn/carEW/gfp were constructed and transformed into E. coli BL21(DE3) strain for the expression of CarEW, CarEW-GFP, and INPN-CarEWGFP fusion proteins during the growth phase, respectively. E. coli BL21(DE3) strain containing the blank vector $\mathrm{pET}-28 \mathrm{a}(+)$ was used as an experimental control. Expression patterns of the fusion proteins were detected by sodium dodecyl sulfate polyacrylamide gel electrophoresis (SDS-PAGE). The results showed that a band corresponding to CarEW at $\sim 53 \mathrm{kDa}$ and $\sim 80 \mathrm{kDa}$ (CarEW, $\sim 53 \mathrm{kDa}$; GFP, $\sim 27 \mathrm{kDa}$; CarEW-GFP, $\sim 80 \mathrm{kDa}$ ) appeared in E. coli BL21(DE3) cells harboring pET-28a(+)/carEW (Fig. 2a, lane 2, indicated by arrow) and pET-28a $(+) / c a r E W / g f p$ (Fig. 2a, lane 3, indicated by arrow), respectively. For E. coli BL21(DE3) cells harboring pET-28a(+)/inpn/carEW/gfp, a band $\sim 80 \mathrm{kDa}$ appeared separately in the cytoplasmic fraction (Fig. 2a, lane 4, indicated by arrow) and outer membrane fraction (Fig. 2a, lane 6, indicated by arrow), while no band was detected in the inner membrane fraction (Fig. 2a, lane 5). No band was detected in the control, E. coli BL21(DE3) cells harboring the blank vector pET-28a(+) (Fig. 2a, Lane 1). These results indicated that the synthesized fusion proteins had been expressed correctly in different cell fractions and the size of the corresponding proteins matched well with the calculated molecular masses.

For the recombinant plasmids, pET$28 \mathrm{a}(+) / c a r E W, \quad$ pET-28a $(+) / c a r E W / g f p$, and pET$28 \mathrm{a}(+) /$ inpn/carEW/gfp, all recombinant proteins were fused with $6 \times$ His tag at the C-terminal of the fusion proteins. The western blot analysis of the expressed proteins using anti-His monoclonal antibody further revealed clear signs of all corresponding proteins from subcellular fractions of $E$. coli BL21(DE3) containing pET28a (+)/carEW (Fig. 2b, lane 2, CarEW, $53 \mathrm{kDa}$ ), E. coli BL21(DE3) containing pET-28a(+)/carEW/gfp (Fig. 2b, lane 3, CarEW-GFP, $\sim 80 \mathrm{kDa}$ ), and E. coli BL21(DE3) containing pET-28a(+)/inpn/carEW/gfp (Fig. 2b, lane 4 and 6 , CarEW-GFP, $80 \mathrm{kDa}$ ), whereas none was detected in the control (Fig. 2b, lane 1).

\section{Surface localization analysis of INPN-CarEW-GFP fusion protein on E. coli cells}

Proteinases cannot cross the outer membrane of the cell, and, therefore, only surface-displayed proteins can be degraded by proteinases [30]. Therefore, evidence for the localization of target proteins on the cell surface can be proved by a proteinase accessibility assay. Proteinase $K$ is a subtilisin-related serine proteinase that exhibits broad substrate specificity and hydrolyzes a variety of peptide bonds. After treatment with proteinase $\mathrm{K}$ for $1 \mathrm{~h}$, the CarEW activity of E. coli BL21(DE3) cells carrying pET-28a $(+) /$ inpn/carEW $\left(\mathrm{OD}_{600}=1.0\right)$ decreased approximately $50 \%$ indicating that the surface-displayed CarEW was degraded by proteinase $\mathrm{K}$, whereas, no obvious activity reduction was observed for $E$. coli BL21(DE3) cells carrying pET-28a(+)/carEW $\left(\mathrm{OD}_{600}=1.0\right)$. This result supported the profile of western blot above that CarEW was approximately half localized on the surface, and half was distributed in the cytoplasmic for the $E$. coli BL21(DE3) cells carrying pET-28a(+)/inpn/carEW plasmid.

To further confirm the presence of INPN-CarEW-GFP fusion protein on the cell surface, green fluorescence was observed. Under fluorescence microscopy, green fluorescence was concentrated at both poles or on membrane for cells containing plasmid pET-28a(+)/inpn/carEW/gfp (Fig. 3b, right panel). However, in the control cells, $E$. coli BL21(DE3) carrying pET-28a(+)/carEW/gfp without INPN anchor protein, the green fluorescence distributed evenly for the whole cells (Fig. 3a, right panel). So, the result suggested that INPN-CarEW-GFP fusion was 


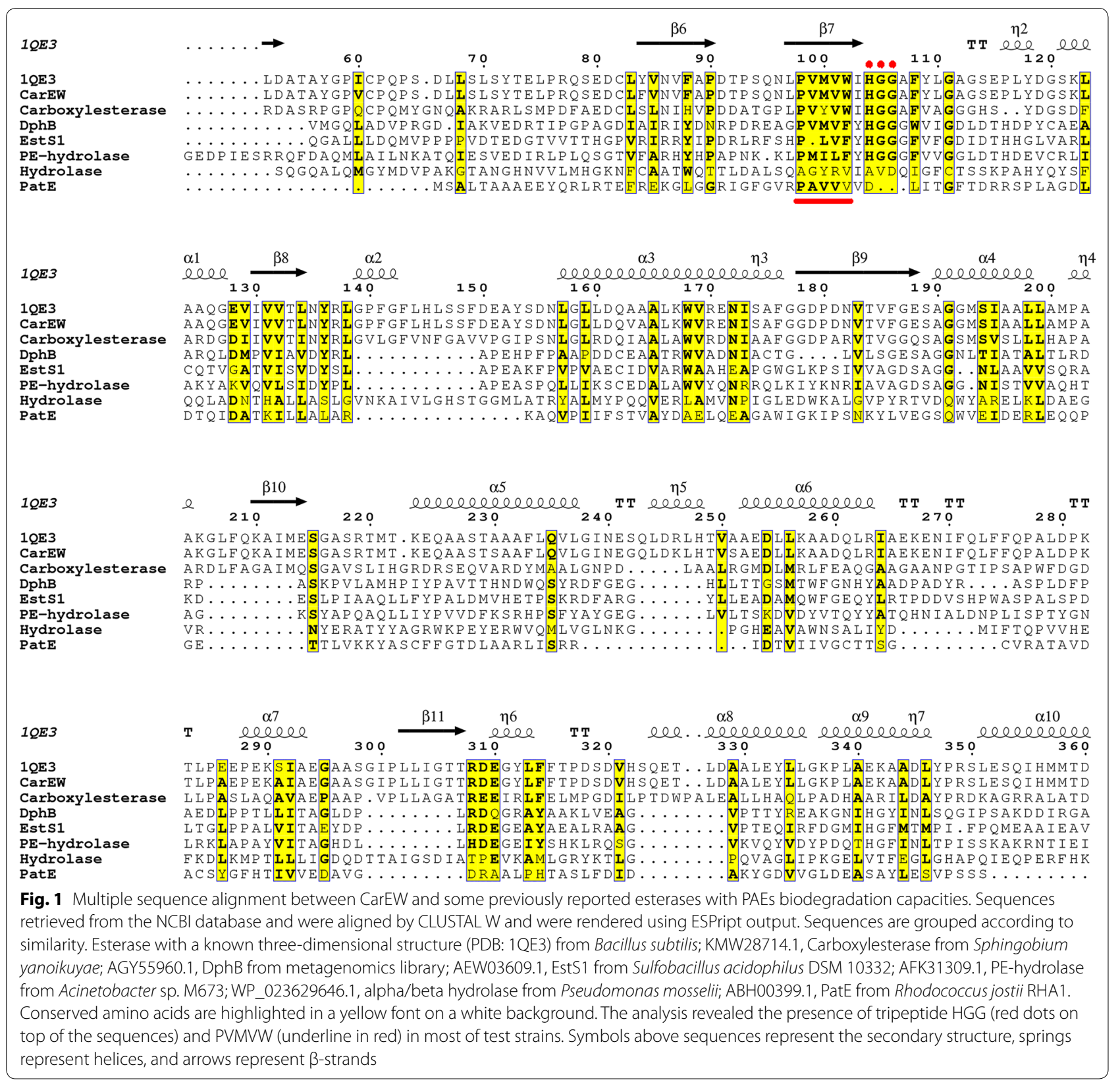

correctly displayed on the surface of $E$. coli BL21(DE3) cells.

\section{Enzymatic activity and stability of E. coli BL21(DE3) strain expressing INPN-CarEW fusion protein}

Enzymatic activity of CarEW was determined as reported previously [10]. The substrate specificity of $E$. coli BL21(DE3) cells displaying CarEW was determined using the same concentrations of various $p$-NP substrates. The engineered $E$. coli cells were active on $p-\mathrm{NPC}_{2}$ to $p-\mathrm{NPC}_{8}$ and displayed maximal enzymatic activity toward $p-\mathrm{NPC}_{2}$
(Additional File 1: Figure S1). The optimal temperature and $\mathrm{pH}$ of the whole cell biocatalyst were investigated. As shown in Fig. 4a, the enzymatic activity increased linearly from $10{ }^{\circ} \mathrm{C}$ to $45{ }^{\circ} \mathrm{C}$ and the maximal activity was detected at $45{ }^{\circ} \mathrm{C}$. More than $40 \%$ of the enzyme activity was observed between 20 and $55^{\circ} \mathrm{C}$, and the whole cell biocatalyst exhibited more than $20 \%$ of the original enzymatic activity when the temperature reached $80{ }^{\circ} \mathrm{C}$. The optimal $\mathrm{pH}$ for the whole cell biocatalyst was determined at $\mathrm{pH} 9.0$ and more than $40 \%$ of enzymatic activity was kept at $\mathrm{pH}$ values ranging from $\mathrm{pH} 6.5$ to 9.0. In addition, 


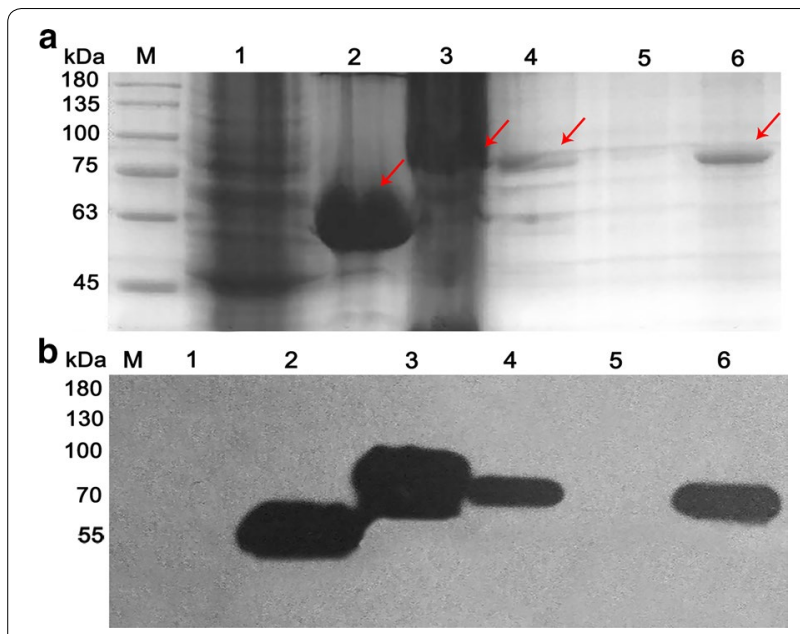

Fig. 2 Expression of recombinant fusion proteins: CarEW, CarEW-GFP and INPN-CarEW-GFP. (a) SDS-PAGE and (b) western blot of lysates of E. coli harboring pET-28a(+) series plasmids. Lane M, protein marker; Lane 1, E. coli cells harboring pET-28a(+); Lane 2, E. coli cells harboring pET-28a(+)/carEW; Lane 3, E. coli cells harboring pET-28a(+)/carEW/gfp; Lane 4, 5, 6, cytoplasmic fraction, inner membrane, and outer membrane of E. coli cells harboring pET-28a( + )/inpn/carEW/gfp, respectively. Anti-His monoclonal antibody was used as a 1:1000 dilution more than $40 \%$ of maximum activity was observed at $\mathrm{pH}$ 10.0 (Fig. 4b). Under both optimal conditions, the enzymatic activity of the whole cell biocatalysts was demonstrated to be a $K_{\text {cat }}$ of $26.46 \pm 0.76 \mathrm{~s}^{-1}$ and $K_{c a t} / K_{m}$ of $833.23 \mathrm{~s}^{-1} \mathrm{mM}^{-1}\left(\mathrm{U} /\right.$ per $\left.\mathrm{OD}_{600}\right)$, respectively.

For surface expression approach, two concerns should be taken into consideration, one is the growth inhibition of cell, and the other is the stability of whole cell biocatalysts. To determine whether the surface display of INPNCarEW fusion inhibits growth of the cell, growth profile of E. coli BL21(DE3) strain carrying pET-28a $(+) /$ carEW or pET-28a $(+) /$ inpn/carEW were compared. Two strain reached almost the same final density after incubated for 2 days, and no growth inhibition was observed for cells expressing INPN-CarEW fusion protein. To investigate the stability, whole cell enzymatic activity of the suspended cultures was determined periodically. The equal volume of engineered whole cell biocatalysts that suspended in citrate- $\mathrm{Na}_{2} \mathrm{HO}_{4}$ buffer $(50 \mathrm{mM}, \mathrm{pH} 9.0)$ at $4{ }^{\circ} \mathrm{C}, 37^{\circ} \mathrm{C}$, and $45^{\circ} \mathrm{C}$, respectively, and residual activity of CarEW was determined intermittently for more than 1 month. No activity decrease of the whole cell biocatalyst was observed, and $200 \%$ of the original enzymatic activity was detected when incubated at $4{ }^{\circ} \mathrm{C}$ for more than 1 month. When the temperature reached $45^{\circ} \mathrm{C}$, the
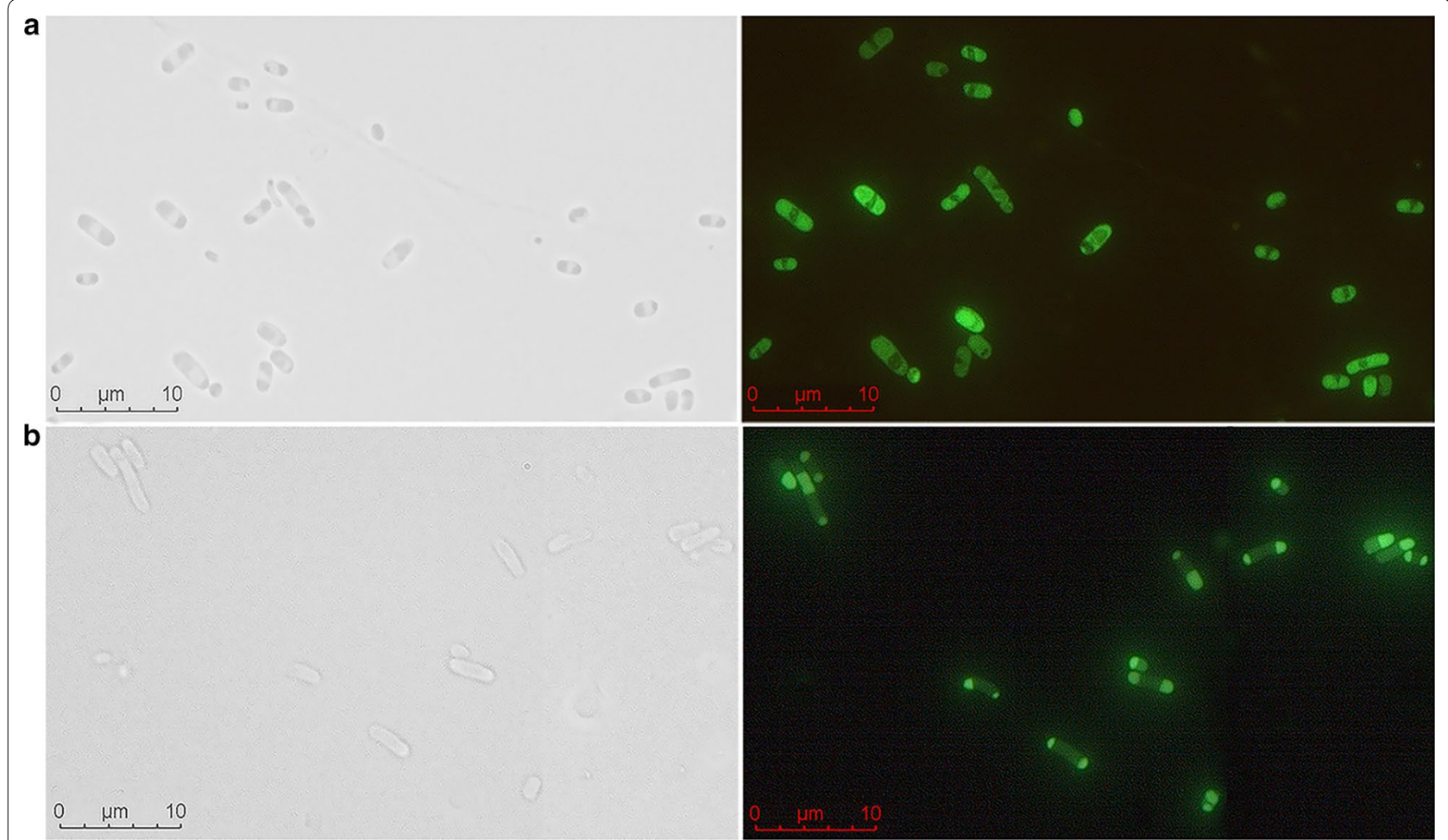

Fig. 3 Fluorescence micrographs of recombinant E. coli BL21 (DE3) strain. a E. coli BL21(DE3) cells carrying pET-28a(+)/carEW/gfp and b pET-28a(+)/inpn/carEW/gfp, respectively. Left panel, microphotographs were taken under visible light; Right panel, fluorescence microphotographs 

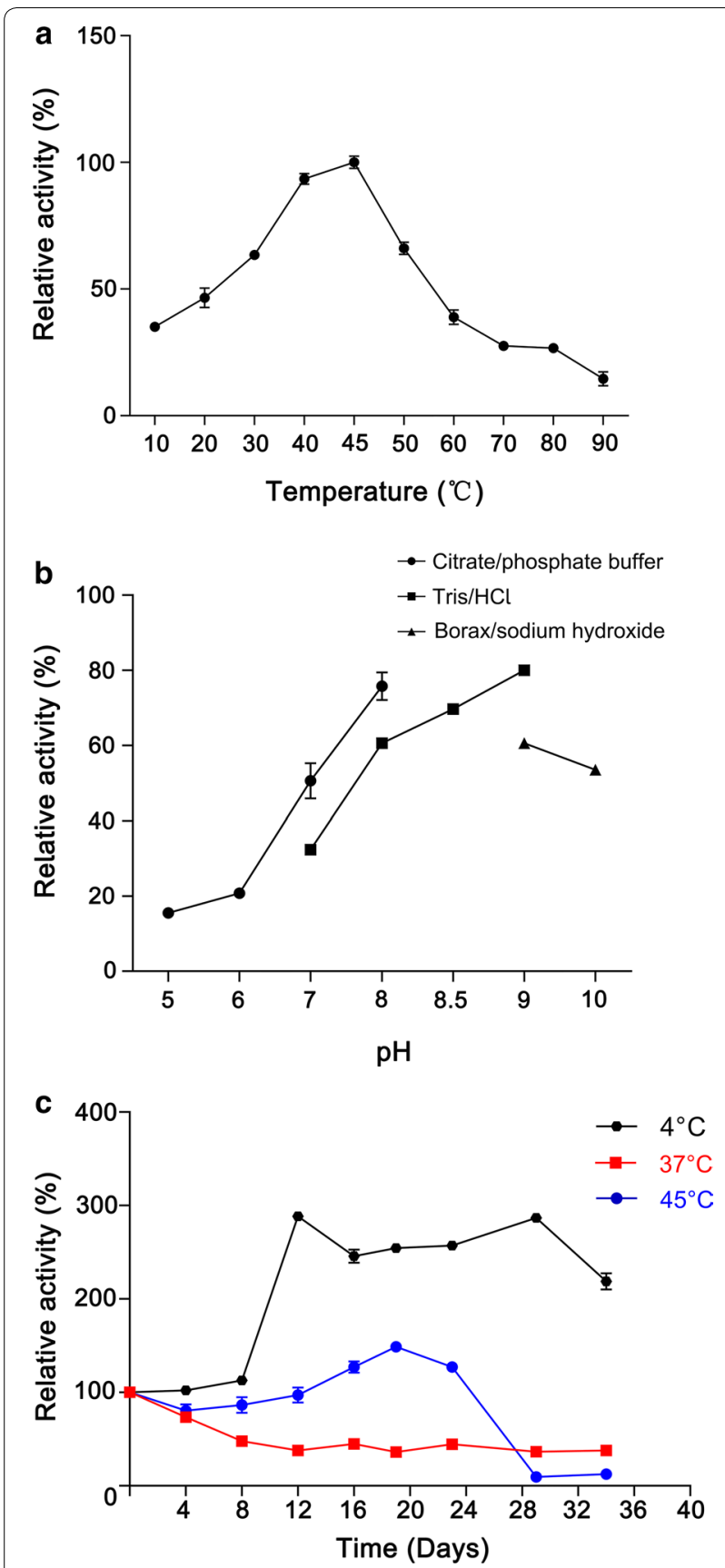

Fig. 4 a The optimum temperature, $\mathbf{b}$ pH, and c Long-term stability of the whole cell biocatalyst. Residual activities were determined periodically for over a month. Values are the means of three replicates \pm the standard deviation

enzymatic activity of engineered cells remained at essentially the original level up until 24th day. Soon after, 90\% of CarEW activity was lost and the activity subsequently fluctuated at around $10 \%$ of the original activity level for the last 10 days. At $37{ }^{\circ} \mathrm{C}$, the E. coli BL21(DE3) strain that surface expressed INPN-CarEW fusion protein

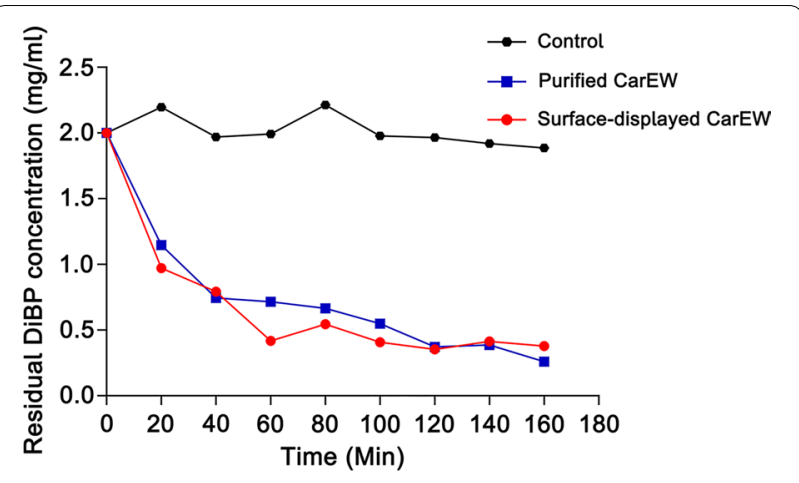

Fig. 5 DiBP degradation by purified CarEW and the whole cell biocatalyst. The error bars represent the mean \pm SD $(n=3)$

exhibited $\sim 50 \%$ of the original activity over 1 month (Fig. 4c). These results illustrated that the surface-displayed INPN-CarEW fusion neither inhibited cell growth nor caused instability of the cell.

\section{Degradation efficiency of DiBP by E. coli BL21(DE3) strain expressing INPN-CarEW fusion protein}

In order to test the degradation efficiency of DiBP by $E$. coli BL21(DE3) strain expressing INPN-CarEW fusion protein, $10 \mathrm{U}$ of whole cell biocatalyst and $10 \mathrm{U}$ of purified CarEW was incubated with $2 \mathrm{mg} / \mathrm{ml} \mathrm{DiBP}$ at $45^{\circ} \mathrm{C}$, respectively. No purified enzyme or whole cell biocatalyst was added in the control. As shown in Fig. 5, $1.5 \mathrm{mg} /$ $\mathrm{ml}$ DiBP was hydrolyzed in 120 min by both biocatalysts, and the biodegradation rate of CarEW surface-displayed cells is a little faster than that of the purified CarEW at $40 \mathrm{~min}$ to $120 \mathrm{~min}$. Therefore, the biodegradation of DiBP was comparable between the whole cell biocatalyst and purified CarEW. However, it is more attractive for the whole cell biocatalyst to be applied in practical use because there are many advantages for the whole cell biocatalyst, such as low cost to obtain, stability and so on.

\section{Discussion}

With the fast development of sequencing technologies, a large number of cultured and uncultured microorganisms were sequenced and their functional genes were annotated. Bacillus sp. K91, a thermophilic bacterium which can grow from 50 to $70{ }^{\circ} \mathrm{C}$, was isolated by our lab from a hot spring water in Teng Chong, Yunnan Province, China. Based on the genome sequencing and annotation, a carboxylesterase, CarEW was cloned and expressed in E. coli BL21(DE3) [10]. Over the past few years, the heterologous proteins have been successfully displayed on the surface of bacteria or fungi, exhibiting promising prospects in many biotechnological processes [25-29]. 
In the present study, CarEW was displayed on the surface of $E$. coli BL21(DE3) using an ice nucleation protein anchor. Using this approach, CarEW on the bacterial surface could be produced and anchored simultaneously. Moreover, this whole cell biocatalyst can be stored and re-used easily, which can be directly used after cultivation and harvest. As shown by the SDS-PAGE (Fig. 2a), western blot (Fig. 2b) and proteinase $\mathrm{K}$ accessibility assay results, approximately $50 \%$ of total CarEW was displayed on the E. coli BL21(DE3) surface, and $~ 50 \%$ of CarEW was expressed in the cytoplasm. Consistent with several other previously reports, less than $50 \%$ target proteins were expressed on the surface of E. coli or in other surface-displaying bacteria, Pseudomonas putida, for example $[31,32]$. Surface display systems mediated by the full length or truncated INP anchors from $P$. syringae have been extensively exploited in E. coli, Pseudomonas sp., and other species. In the future, other anchors, such as outer membrane protein A (OmpA), OmpC, or OmpF, and so on, and other surface-displayed microorganisms, Saccharomyces cerevisiae, for example, are deserved to be determined [33, 34]. In addition, the green fluorescence was concentrated at both poles or on outer membrane of E. coli BL21(DE3) strain carrying pET-28a(+)/inpn/ carEW/gfp plasmid, while GFP distributed evenly for E. coli BL21(DE3) strain carrying $\mathrm{pET}-28 \mathrm{a}(+) /$ carEW/ $g f p$ plasmid, without the INP anchor motif (Fig. 3). This result observed here is consistent with other previous reports using the same INP-mediated system [35]. As mentioned above, about $50 \%$ of target proteins can be displayed on the surface of cells when used the INPmediated surface-displayed system, as also observed by CarEW. Therefore, we supposed that the GFP might be buried in the cell wall and not successfully displayed on the surface, then lead to the GFP signal was concentrated at both pores for cells carrying pET-28a $(+) /$ inpn/carEW/ $g f p$ plasmid. These might also explain that the activity of whole cell biocatalyst increased at the 12th day might be due to the release of CarEW resided in the cytoplasm caused by cell lysis, which was similar to an organophosphorus hydrolase using the same surface display system [27].

The use of engineered microorganisms as bioremediating biocatalysts to eliminate pollutants in the environment represents a promising strategy [27, 31]. In this present study, a laboratory-scale whole cell biocatalyst used for DiBP degradation was developed, and a schematic diagram represented the progress was constructed (Fig. 6). To the best of our knowledge, this work is the first approach to degrade hazardous DiBP using engineered bacterial cells with surface-displayed carboxylesterase. In the natural environment, many factors influence the biodegradation efficiencies, such as the fluctuating environmental conditions, different microbial populations, complex contaminants, and so on. Therefore, although the biodegradation efficiency of $\mathrm{DiBP}$ is comparable between the purified CarEW and CarEW whole cell biocatalyst, the stability (stable at $4{ }^{\circ} \mathrm{C}$ and $45{ }^{\circ} \mathrm{C}$ ) (Fig. 4c) and availability made the whole cell biocatalyst better suitable for practical environmental bioremediation. Additionally, there are usually a variety of contaminants exist at a one single site in most situations, thus, the application of this CarEW surface-displayed engineered strain for removal of PAEs besides DiBP need to be further investigated.

\section{Conclusions}

Here, a surface displayed system based on INPN as a carrier protein for DiBP biodegradation was developed. The INPN-CarEW surface display fusion protein had no negative effect on cell growth or membrane integrity. This engineered strain had the capacity to degrade DiBP, which emphasizes high potential to use this strain for removal of other kinds of PAEs pollutants in the environment or use this strategy to develop other bioremediation approaches.

\section{Methods}

\section{Substrates, reagents and plasmids}

$p$-NP esters with various carbon chain lengths $\left(p-\mathrm{NPC}_{2}\right.$ to $p$-NPC ${ }_{16}$ ) were purchased from Sigma-Aldrich (USA) or TCI (Tokyo, Japan). Diisobutyl phthalate (DiBP), dibutyl phthalate (DBP), Bis (2-ethylhexyl) phthalate (DEHP), and diethyl phthalate (DEP) were purchased from $J \& K$ Scientific Ltd., China. pEASY-E2 expression kit and fast $p f u$ DNA polymerase were provided by TransGen Biotech (Beijing, China). Escherichia coli BL21(DE3) and pET-28a $(+)$ expression vector was from Novagen (USA). Qiagen provided the Nickel-NTA agarose (Germany). pEGFP-N3, pMD18-T and restriction enzymes BamHI and HindIII were purchased from Takara. Luria-Bertani (LB) bacteria growth medium was obtained from Thermo Fisher Scientific (USA). All other chemicals were at least analytical grade and were obtained from Sigma (USA) or Sinopharm Chemical Reagent (Shanghai, China).

\section{Plasmid construction}

The inak gene was biosynthesized by Sangon Biotech, Shanghai, China, and subcloned into pMD18-T to generate pMD18-T/inak. To construct pET-28a $(+) /$ carEW, carEW gene was amplified using primers $\mathrm{P} 1$ and $\mathrm{P} 2$ from a previously constructed plasmid, pEASY-E2/carEW [10]. The PCR products were digested with $B a m H I$ and HindIII, and ligated with similarly digested pET-28a $(+)$ to generate $\mathrm{pET}-28 \mathrm{a}(+) / c a r E W$. To construct pET$28 \mathrm{a}(+) / c a r E W / g f p, g f p$ gene was amplified with primers 


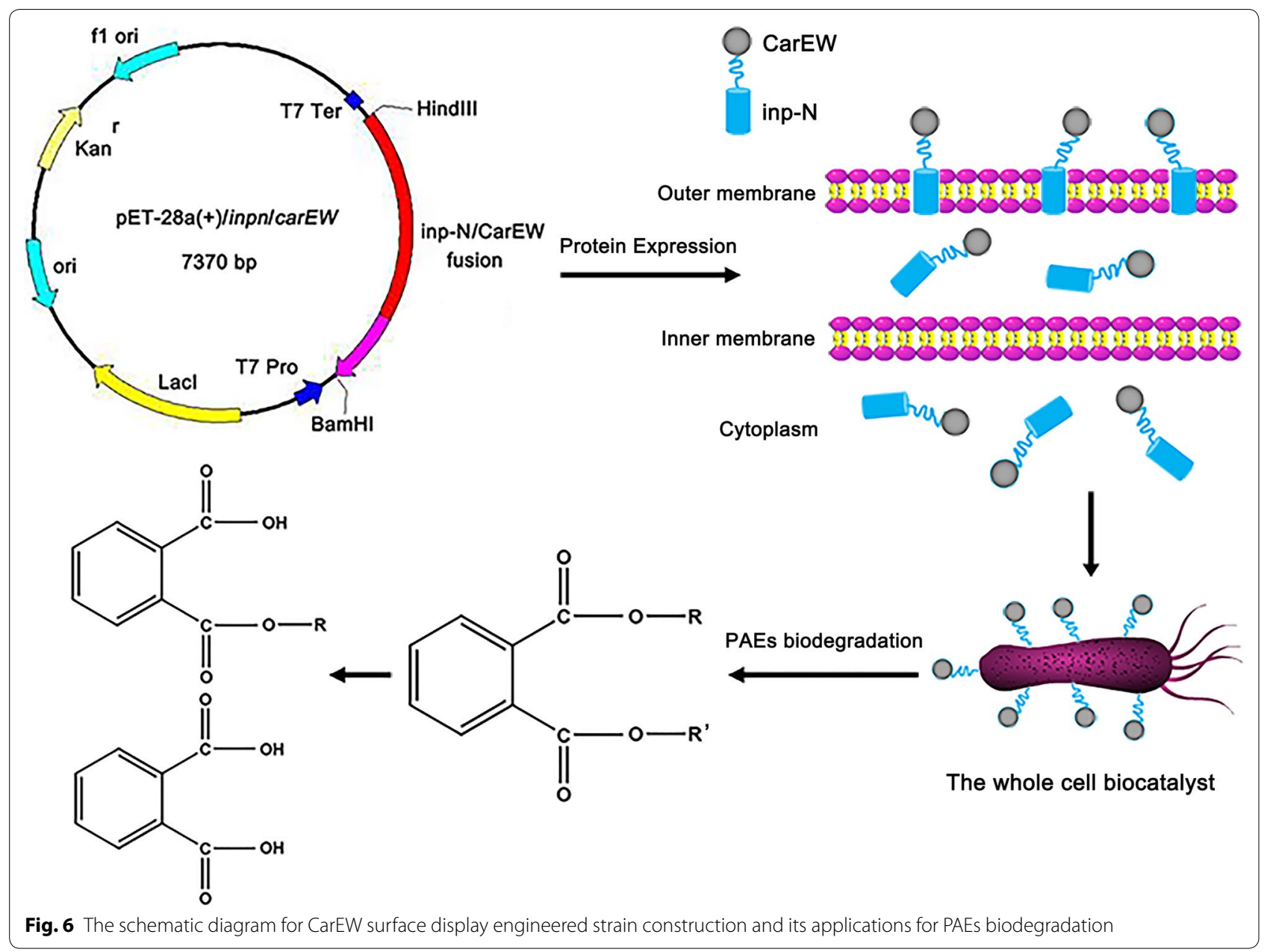

P3 and P4 using pEGFP-N3 as a template, and ligated to similarly HindIII digested pET-28a $(+) / c a r E W$. For the construction of $\mathrm{pET}-28 \mathrm{a}(+) /$ inpn/carEW, the N-terminal domain of inak gene (named as inpn gene) was amplified from plasmid pMD18-T/inak with primers P5 and P6, and then was ligated to similarly BamHI digested pET$28 \mathrm{a}(+) /$ carEW. To construct $\mathrm{pET}-28 \mathrm{a}(+) /$ inpn/carEW/ $g f p, g f p$ gene was amplified with primers P7 and P8, and the PCR products were ligated to similarly BamHI digested pET-28a(+)/inpn/carEW. All the constructed recombinant plasmids were confirmed by sequencing (Sangon Biotech, Shanghai, China). A schematic diagram for the construction of recombinant plasmids are shown in Additional file 2: Figure S2. Primers and plasmids used in this study are summarized in Additional file 3: Table S1.

\section{Enzyme activity of surface-displayed CarEW}

Esterase activity was determined at $405 \mathrm{~nm}$ by measuring the absorbance of liberated $p$-NP as reported previously [10]. The amount of enzyme required to release $1 \mu \mathrm{M} p$-NP per minute was defined as one unit of enzyme activity (U). Different buffers $(50 \mathrm{mM})$ : citrate/phosphate buffer $(\mathrm{pH} 5.0-8.0), \quad$ Tris/ $\mathrm{HCl}(\mathrm{pH}$ 8.0-9.0), and boric acid/borax ( $\mathrm{pH}$ 9.0-10.0) were used to determine the optimal $\mathrm{pH}$ of surface-displayed CarEW. Temperatures ranging from 0 to $80{ }^{\circ} \mathrm{C}$ was used to determine optimal temperature at $\mathrm{pH} 9.0$ using $p$ $\mathrm{NPC}_{2}$ as substrate. Substrate specificity were investigated using different $p$-NP esters $\left(p-\mathrm{NPC}_{2}\right.$ to $p$ - $\left.\mathrm{NPC}_{16}\right)$. Kinetic was determined using different concentrations of $p-\mathrm{NPC}_{2}(0.12$ to $1.2 \mathrm{mM})$ at $45^{\circ} \mathrm{C}$ and $\mathrm{pH}$ 9.0. The Michaelis-Menten constant $\left(K_{m}\right)$ and maximum velocity $\left(V_{\max }\right)$ were investigated by a nonlinear regression method.

To determine the stability of whole cell biocatalyst, E. coli BL21 cells harboring $\mathrm{pET}-28 \mathrm{a}(+) /$ inpn/carEW were resuspended in $50 \mathrm{mM}$ Tris $/ \mathrm{HCl}$ buffer $(\mathrm{pH} 9.0)$ after induction and incubated at $4{ }^{\circ} \mathrm{C}, 37^{\circ} \mathrm{C}$, and $45{ }^{\circ} \mathrm{C}$, respectively. An identical volume of sample solution was extracted at regular intervals for over a month to facilitate CarEW activity determination. 


\section{Cell fractionation, western blot and proteinase $\mathrm{K}$ accessibility assay}

The pET-28a $(+)$ series plasmids including pET-28a $(+)$, $\mathrm{pET}-28 \mathrm{a}(+) /$ carEW, $\mathrm{pET}-28 \mathrm{a}(+) /$ inpn/carEW, pET$28 \mathrm{a}(+) / c a r E W / g f p$, and $\mathrm{pET}-28 \mathrm{a}(+) / i n p n / c a r E W / g f p$ were transformed into $E$. coli BL21(DE3) cells and were cultivated in LB medium supplemented with $100 \mu \mathrm{g} /$ $\mathrm{ml}$ ampicillin at $37{ }^{\circ} \mathrm{C}$, separately. $0.1 \mathrm{mM}$ of Isopropyl $\beta$-D-1-thiogalactopyranoside (IPTG) was added when $\mathrm{OD}_{600}$ reached 0.5 , respectively. Then, the cell cultures were maintained at $20{ }^{\circ} \mathrm{C}$ for another $20 \mathrm{~h}$ for protein induction. After harvested $\left(12,000 \times g\right.$ for $\left.30 \mathrm{~min}, 4{ }^{\circ} \mathrm{C}\right)$ and resuspended in PBS buffer ( $\mathrm{pH} 7.0$ ), cell pellets were disrupted by sonication $(7 \mathrm{~s}, 150 \mathrm{w})$ for $15 \mathrm{~min}$ on ice, and the supernatant was collected $(12,000 \times g$ for $\left.30 \mathrm{~min}, 4^{\circ} \mathrm{C}\right)$. Total membrane fractions were obtained by centrifugation at $37,000 \mathrm{rpm}$ for $4 \mathrm{~h}$ (at $4{ }^{\circ} \mathrm{C}$ ). Inner membrane was solubilized by resuspended the total membrane in PBS containing $0.01 \mathrm{mM} \mathrm{MgCl}_{2}$ and $2 \%$ Triton X-100 and at room temperature (RT) for $30 \mathrm{~min}$. Subsequently, the outer membrane fraction was re-pelleted by centrifugation at $37,000 \mathrm{rpm}$ for $4 \mathrm{~h}$ (at $4{ }^{\circ} \mathrm{C}$ ), and the inner membrane remained in the supernatant. For purification of CarEW, E. coli BL21(DE3) cells containing pET-28a(+)/carEW plasmid were collected, disrupted and the supernatant was applied to a $\mathrm{Ni}^{2+}-\mathrm{NTA}$ agarose. Protein concentration was determined by the Bradford method using bovine serum albumin as standard [36].

For western blot analysis, cell-free extracts (crude extracts) and different cell fractions $(\sim 10 \mathrm{mg} / \mathrm{ml}$ of total protein of each sample) were separated by SDSPAGE (12\%), and then proteins were transferred (transfer buffer: $192 \mathrm{mM}$ glycine, $25 \mathrm{mM}$ Tris base, and 20\% methanol, $\mathrm{pH}$ 8.0) onto a polyvinylidene difluoride (PVDF) membrane (Millipore, USA). Monoclonal Histag antibody (IgG2), peroxidase-conjugated goat antimouse IgG $(\mathrm{H}+\mathrm{L})$ (both obtained from ZSGB-BIO, China), and a Super Signal West Pico kit from Thermo Scientific Pierce (USA) were used, and procedures were conducted following the method as reported by Nguyen et al. [26].

To investigate surface exposure of the CarEW, the proteinase $\mathrm{K}$ accessibility test was used. E. coli BL21(DE3) cells harboring pET-28a(+)/inpn/carEW $\left(\mathrm{OD}_{600}=1.0\right)$ were incubated in PBS buffer ( $\left.\mathrm{pH} 7.0\right)$ with $100 \mu \mathrm{g} / \mathrm{ml}$ proteinase $\mathrm{K}$ (Sigma, USA) at $37^{\circ} \mathrm{C}$ for $1 \mathrm{~h}$, and the digest was terminated by adding $10 \mu \mathrm{M}$ of phenylmethylsulfonylfluoride (PMSF) (Sigma, USA) following incubation on ice for $5 \mathrm{~min}$. The proteinase K-treated and untreated cells were assayed for CarEW activity as described above.

\section{Fluorescence microscopic assay}

After harvested and washed three times with PBS (pH 7.0), E. coli BL21(DE3) cells harboring $\mathrm{pET}-28 \mathrm{a}(+) /$ inpn/carEW/gfp plasmid and pET$28 \mathrm{a}(+) /$ carEW/gfp plasmid were diluted to $\mathrm{OD}_{600} 1.0$, respectively. The green fluorescence was observed using LEICA DM6 B (Leica DMB, Wetzlar, Germany) with a 100-oil immersion objective and photographed with an N21 (BP 515-560) filter.

\section{Degradation of DiBP by cell surface-displayed CarEW}

A $1-\mathrm{ml}$ reaction mixture containing $10 \mathrm{U}$ of surfacedisplayed CarEW or purified CarEW and $2 \mathrm{mg} / \mathrm{ml}$ DiBP (solubilized in $50 \mu \mathrm{l}$ dimethyl sulfoxide (DMSO)) in $50 \mathrm{mM}$ Tris $/ \mathrm{HCl}(\mathrm{pH} 9.0)$ was incubated at $45^{\circ} \mathrm{C}$. The reaction was terminated at indicated time points $(0,20,40,60,80,100,120,140,160,180 \mathrm{~min})$ with $10 \%$ (v/v) $1 \mathrm{~N} \mathrm{HCl}$, and equal volume of ethyl acetate was used to extract reaction products. The extracts were redissolved in $1 \mathrm{ml}$ of methanol after dried over $\mathrm{Na}_{2} \mathrm{SO}_{4}$, and the residual DiBP was analyzed on an Agilent gas chromatography-mass spectrometry (GC-MS) system (HP6890-5973 N).

The GC capillary column used was HP-5MS (0.25 mm by $0.25 \mu \mathrm{m}$ by $30 \mathrm{~m}$ ). The programmed oven temperature was as following: initial temperature of $60{ }^{\circ} \mathrm{C}$ for $1 \mathrm{~min}$, followed by a $20^{\circ} \mathrm{C} \mathrm{min}^{-1}$ increase to $220^{\circ} \mathrm{C}$ and maintained for $1 \mathrm{~min}$; this was followed by a $5{ }^{\circ} \mathrm{C} \mathrm{min}{ }^{-1}$ increase to $250{ }^{\circ} \mathrm{C}$ and maintained for $1 \mathrm{~min}$. This was followed by a $20{ }^{\circ} \mathrm{C} \mathrm{min} \mathrm{min}^{-1}$ increase to $290{ }^{\circ} \mathrm{C}$ and was maintained for $7.5 \mathrm{~min}$. The injector temperature was set to $260{ }^{\circ} \mathrm{C}$ and helium was used with a constant column flow rate of $1 \mathrm{ml} \mathrm{min}^{-1}$. One microliter of each sample extract was injected in splitless mode. Controls without enzyme were analyzed in parallel.

\section{Supplementary information}

Supplementary information accompanies this paper at https://doi. org/10.1186/s12934-020-01373-6.

Additional file 1: Figure S1. Substrate specificity of E. coli BL21(DE3) displaying INPN-CarEW fusion protein towards acetylated esters with different lengths.

Additional file 2: Figure S2. Schematic diagram of the procedure to construct recombinant plasmids for bacterial cell surface display.

Additional file 3: Table S1. Plasmids and primers used in this study.

\section{Authors' contributions}

JD and ZH conceived of the study; $Y Z, C W$, and ZP designed and performed experiments; JD, YZ, and CW analyzed data. JD and CW wrote the manuscript. YM and XT provided resources of experiment. All authors read and approved the final manuscript.

\section{Funding}

This work was supported by the National Natural Science Foundation of China (Grant No. 31960023, 31600083); the Reserve Talents Project for Young and 
Middle-Aged Academic and Technical Leaders of Yunnan Province (Grant No. 2018HB059), and the National Key Research and Development Program of China (Grant No. 2017YFB0308401).

\section{Availability of data and materials}

All data generated or analyzed during this study are included in this published article and its additional files.

\section{Ethics approval and consent to participate}

Not applicable.

\section{Consent for publication}

Not applicable.

\section{Competing interests}

The authors declare that they have no competing interests.

\begin{abstract}
Author details
${ }^{1}$ Engineering Research Center of Sustainable Development and Utilization of Biomass Energy, Ministry of Education, Yunnan Normal University, Kunming 650500, Yunnan, China. ${ }^{2}$ Key Laboratory of Yunnan for Biomass Energy and Biotechnology of Environment, Kunming 650500, Yunnan, China. ${ }^{3}$ Key Laboratory of Enzyme Engineering, Yunnan Normal University, Kunming 650500, Yunnan, China.
\end{abstract}

Received: 10 February 2020 Accepted: 25 May 2020

Published online: 29 May 2020

\section{References}

1. Net S, Sempere R, Delmont A, Paluselli A, Ouddane B. Occurrence, fate, behavior and ecotoxicological state of phthalates in different environmental matrices. Environ Sci Technol. 2015;49:4019-35.

2. Gao DW, Li Z, Wang H, Liang H. An overview of phthalate acid ester pollution in China over the last decade: environmental occurrence and human exposure. Sci Total Environ. 2018;645:1400-9.

3. Bui TT, Giovanoulis G, Cousins AP, Magnér J, Cousins IT, de Wit CA. Human exposure, hazard and risk of alternative plasticizers to phthalate esters. Sci Total Environ. 2016;541:451-67.

4. Weir SM, Wooten KJ, Smith PN, Salice CJ. Phthalate ester leachates in aquatic mesocosms: implications for ecotoxicity studies of endocrine disrupting compounds. Chemosphere. 2014;103:44-50.

5. Mu D, Gao FM, Fan ZL, Shen H, Peng H, Hu JY. Levels of phthalate metabolites in urine of pregnant women and risk of clinical pregnancy loss. Environ Sci Technol. 2015:49:10651-7.

6. Katsikantami I, Sifakis S, Tzatzarakis MN, Vakonaki E, Kalantzi OI, Tsatsakis AM, et al. A global assessment of phthalates burden and related links to health effects. Environ Int. 2016;97:212-36.

7. Yost EE, Euling SY, Weaver JA, Beverly BEJ, Keshava N, Mudipalli A, et al. Hazards of diisobutyl phthalate (DIBP) exposure: a systematic review of animal toxicoloy studies. Environ Int. 2019;125:579-94.

8. Mahajan R, Verma S, Kushwaha M, Singh D, Akhter Y, Chatterjee S. Biodegradation of dinbutyl phthalate by psychrotolerant Sphingobium yanoikuyae strain P4 and protein structural analysis of carboxylesterase involved in the pathway. Int J Biol Macromol. 2019;122:806-16.

9. Singh N, Dalal V, Mahto JK, Kumar P. Biodegradation of phthalic acid esters (PAEs) and in silico structural characterization of mono-2-ethylhexyl phthalate (MEHP) hydrolase on the basis of close structural homolog. J Hazard Mater. 2017;338:11-22.

10. Ding JM, Wang CF, Xie ZR, Li JJ, Yang YJ, Mu YL. Properties of a newly identified esterase from Bacillus sp. K91 and its novel function in diisobutyl phthalate degradation. PLOS ONE. 2015;10:e0119216.

11. Zhang XY, Fan X, Qiu YJ, Li CY, Xing S, Zheng YT, et al. Newly identified thermostable esterase from Sulfobacillus acidophilus: properties and performance in phthalate ester degradation. Appl Microbiol Biotechnol. 2014;80:6870-8.

12. Wu J, Liao X, Yu F, Wei Z, Yang L. Cloning of a dibutyl phthalate hydrolase gene from Acinetobacter sp. strain M673 and functional analysis of its expression product in Escherichia coli. Appl Microbiol Biotechnol. 2013;97:2483-91.
13. Hara H, Stewart GR, Mohn WW. Involvement of a novel ABC transporter and monoalkyl phthalate ester hydrolase in phthalate ester catabolism by Rhodococcus jostii RHA1. Appl Environ Microbiol. 2010;76:1516-23.

14. Luo ZH, Wu YR, Chow RKK, Luo JJ, Gu JD, Vrijmoed LLP. Purification and characterization of an intracellular esterase from a Fusarium species capable of degrading dimethyl terephthalate. Process Biochem. 2012;47:687-93.

15. Ahn JY, Kim YH, Min J, Lee J. Accelerated degradation of dipentyl phthalate by Fusarium oxysporum f. sp. pisi cutinase and toxicity evaluation of its degradation products using bioluminescent bacteria. Curr Microbiol. 2006;52:340-4.

16. Nishioka T, Iwata M, Imaoka T, Mutoh M, Egashira Y, Nishiyama T, et al. A mono-2-ethylhexyl phthalate hydrolase from a Gordonia sp. that is able to dissimilate di-2-ethylhexyl phthalate. Appl Environ Microbiol. 2006;72:2394-9.

17. Maruyama K, Akita K, Naitou C, Yoshida M, Kitamura T. Purification and characterization of an esterase hydrolyzing monoalkyl phthalates from Micrococcus sp. YGJ1. Biosci Biotechnol Biochem. 2005;65:1680-3.

18. Saito T, Hong P, Tanabe R, Nagai K, Kato K. Enzymatic hydrolysis of structurally diverse phthalic acid esters by porcine and bovine pancreatic cholesterol esterases. Chemosphere. 2010;81:1544-8.

19. Jiao Y, Chen X, Wang X, Liao X, Xiao L, Miao A, et al. Identification and characterization of a cold-active phthalate esters hydrolase by screening a metagenomic library derived from biofilms of a wastewater treatment plant. PLOS ONE. 2013;8:e75977.

20. Ren L, Lin Z, Liu HM, Hu HQ. Bacteria-mediated phthalic acid esters degradation and related molecular mechanisms. Appl Microbiol Biotechnol. 2018;102:1085-96.

21. Zang HL, Wang HL, Miao L, Cheng Y, Zhang YT, Liu Y, et al. Carboxylesterase, a de-esterification enzyme, catalyzes the degradation of chlorimuron-ethyl in Rhodococcus erythropolis D310-1. J Hazard Mater. 2020;387:121684-94.

22. Bai LS, Zhao CX, Xu JJ, Feng C, Li YQ, Dong YL, et al. Identification and biochemical characterization of carboxylesterase $001 \mathrm{G}$ associated with insecticide detoxification in Helicoverpa armigera. Pestic Biochem Phys. 2019;157:69-79.

23. Hatfield MJ, Umans RA, Hyatt JL, Edwards CC, Wierdl M, Tsurkan L, et al. Carboxylesterase: general detoxifying enzymes. Chem Biol Interact. 2016;259:327-31.

24. Nakamura AM, Kadowaki M, de Godoy A, Nascimento A, Polikarpov I. Low-resolution envelope, biophysical analysis and biochemical characterization of a short-chain specific and halotolerant carboxylesterase from Bacillus licheniformis. Int J Biol Macromol. 2018;120:1893-905.

25. van Bloois E, Winter RT, Kolmar H, Fraaije MW. Decorating microbes: surface display of proteins on Escherichia coli. Trends Biotechnol. 2011:29:79-86.

26. Nguyen HM, Pham ML, Stelzer EM, Plattner E, Grabherr R, Mathiesen $\mathrm{G}$, et al. Constitutive expression and cell-surface display of a bacterial ß-mannanase in Lactobacillus plantarum. Microb Cell Fact. 2019;18:76.

27. Tang XJ, Liang B, Yi TY, Manco G, Palchetti I, Liu AH. Cell surface display of organophosphorus hydrolase for sensitive spectrophotometric detection of p-nitrophenol substituted organophosphates. Enzyme Microb Technol. 2014:55:107-12.

28. Yang CE, Chu IM, Wei YH, Tsai SL. Surface display of synthetic phytochelatins on Saccharomyces cerevisiae for enhanced ethanol production in heavy metal-contaminated substrates. Bioresour Technol. 2017:245:1455-60.

29. Guo Q, An YF, Yun JH, Yang MM, Magocha TA, Zhu JF, et al. Enhanced d-tagatose produdction by spore surface-displayed L-arabinose isomerase from isolated Lactobacillus Brevis PC16 and biotransformation. Bioresour Technol. 2018;247:940-6.

30. Yang C, Liu RH, Yuan YL, Liu JL, Cao XY, Qiao CL, et al. Construction of a green fluorescent protein (GFP)-marked multifunctional pesticidedegrading bacterium for simultaneous degradation of organophosphates and r-hexachlorocyclohexane. J Agr Food Chem. 2013;61:1328-34.

31. Wang W, Zhang Z, Ni H, Yang XM, Li QQ, Li L. Decolorization of industrial synthetic dyes using engineered Pseudomonas putida cells with surfaceimmobilized bacterial laccase. Microb Cell Fact. 2012;11:75.

32. Li Q, Yu Z, Shao X, He J, Li L. Improved phosphate biosorption by bacterial surface display of phosphate-binding protein utilizing ice nucleation protein. FEMS Microbiol Lett. 2009;299:44-52. 
33. Chen TT, Wang KH, Chi X, Zhou LZ, Li JJ, Liu LQ, et al. Construction of a bacterial surface display system based on outer membrane protein $\mathrm{F}$. Microb Cell Fact. 2019;18:70

34. Álvarez-Cao ME, Cerdán ME, González-Siso MI, Becerra M. Optimization of Saccharomyces cerevisiae a-galactosidase production and application in the degradation of raffinose family oligosaccharides. Microb Cell Fact. 2019;18:172.

35. Tatyana R, Andrew RB, Jenifer LH, Boris M, Janet MW. Protein localization in Escherichia coli cells: comparison of the cytoplasmic membrane proteins ProP, LacY, ProW, AqpZ, MscS, and MscL. J Bacteriol. 2010;192:912-24
36. Bradford MM. A rapid and sensitive method for quantitation of microgram quantities of protein utilizing the principle of protein-dye binding Anal Biochem. 1976:72:248-54.

\section{Publisher's Note}

Springer Nature remains neutral with regard to jurisdictional claims in published maps and institutional affiliations.
Ready to submit your research? Choose BMC and benefit from:

- fast, convenient online submission

- thorough peer review by experienced researchers in your field

- rapid publication on acceptance

- support for research data, including large and complex data types

- gold Open Access which fosters wider collaboration and increased citations

- maximum visibility for your research: over $100 \mathrm{M}$ website views per year

At BMC, research is always in progress.

Learn more biomedcentral.com/submissions 